\title{
Maik A. Jochmann and Torsten C. Schmidt: Compound-specific stable isotope analysis
}

\author{
Thomas B. Hofstetter
}

Published online: 25 January 2013

(C) Springer-Verlag Berlin Heidelberg 2013

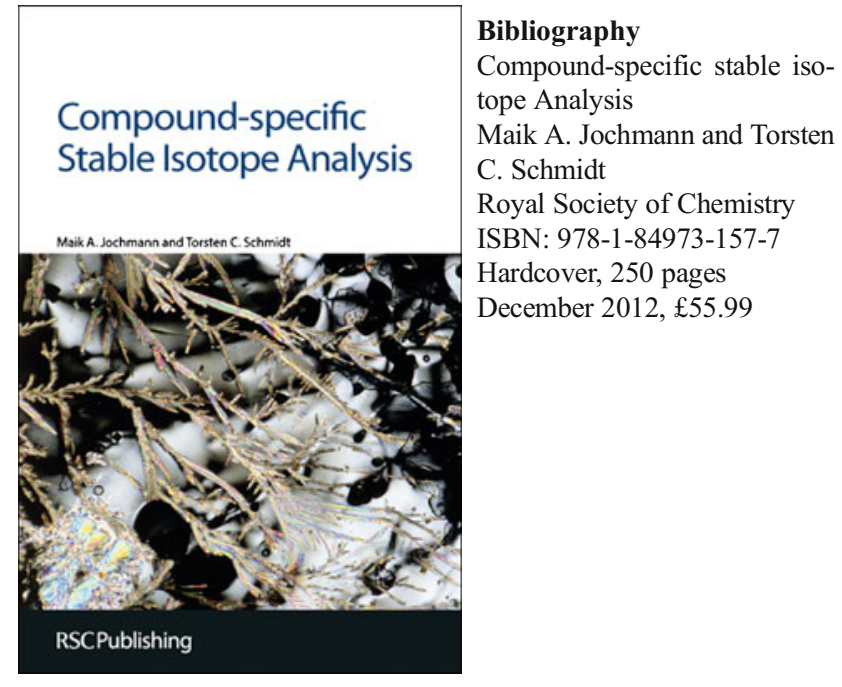

Books' topic The analysis of stable isotope ratios in individual compounds is becoming an established method in the analytical chemist's toolbox. The on-line coupling of chromatographic separation and isotope ratio mass spectrometry makes it possible to identify sources and precursor materials of a target compound from isotopic fingerprints even if it is present in a complex matrix. The raising number of application of compound-specific stable isotope analysis (CSIA), for example, in (environmental) forensics, doping control, and food authentication is evidence for its increasing importance. CSIA also offers novel avenues to track, for example, transformation processes of organic groundwater contaminants, by making use of systematic variation of stable isotope compositions associated with (bio)chemical reactions. However, CSIA requires dedicated instrumentation, a lot of expert

\footnotetext{
T. B. Hofstetter $(\square)$

Environmental Chemistry, Eawag, Überlandstrasse 133,

8600 Dübendorf, Switzerland

e-mail: Thomas.Hofstetter@eawag.ch
}

knowledge, and often years of hands-on experience. The textbook-like companion by Jochmann and Schmidt will make this method more accessible and the book is a welcome promotion for a promising analytical tool.

Contents The art of CSIA is described in seven chapters. In the introductory one, the authors briefly summarize historical and instrumental developments that have led to the stateof-the art and they make the important distinction between bulk and compound-specific techniques.

Chapter two is devoted to basic concepts that are applied throughout the book. A short excursion to the structure of atoms is made to define essential isotope-related terms in physical chemistry. Following the latest IUPAC definitions, expressions are given for isotope-amount fractions, isotope ratios, and the $\delta$ scale with which isotope distributions at natural abundances are quantified. Terms and expressions are exemplified for the elements covered in this book, namely $\mathrm{H}, \mathrm{C}, \mathrm{N}, \mathrm{O}, \mathrm{S}, \mathrm{Cl}$, and $\mathrm{Br}$. The chapter closes with derivations for the interpretation of kinetic and equilibrium isotope fractionation.

Chapters 3 to 5 are the core part of the book and contain a wealth of practical information. The authors thoroughly guide readers through the essential parts of an isotope ratio mass spectrometer and its inlet systems before turning to the coupling to gas and liquid chromatography (Chapter 3). Some essential aspects of chromatography with particular relevance for isotopic analyses are reviewed here. Chapter 4 is devoted to sample preparation and includes a discussion of strategies for organic compound derivatization. Chapter 5 deals with calibration, referencing, and normalization of isotope ratio data and provides practical hints for quality assurance in stable isotope laboratories.

A large selection of illustrative applications of CSIA is given in Chapter 6. Following a short introduction to current challenges in the fields of authenticity control of food and beverages, forensics, archaeology, doping control, environmental sciences, and extraterrestrial matter, 
the authors illustrate the important contributions that isotopic analysis in individual compounds can make.

The book ends with a summary of ongoing analytical developments. Each chapter is accompanied with an extensive list of references and a selection of links to electronic illustrative materials.

Comparison with existing literature This book is dedicated to analytical approaches to isotope ratio measurements and focuses almost exclusively on the isotopic analyses in individual compounds after their chromatographic separation. With this strategy, the authors follow an alternative path to stable isotope analysis and research. Many other monographs in the field deal with isotopes and isotopically substituted molecules in the context of a scientific discipline or field of application, for example, in chemistry (Wolfsberg et al., Isotope Effects in Chemical, Geological, and Bio Sciences 2010), ecology (Dawson \& Siegwolf (Eds.), Stable Isotopes as Indicators of Ecological Change 2007), and forensics (MeierAugenstein, Stable Isotope Forensics 2010) to name just a few. In this context, instrumental aspects of isotope analyses are usually of minor concern or not focussed on CSIA. The latter is covered in detail in handbooks (e.g., de Groot (Ed.), Handbook of stable isotope analytical techniques 2004/2009) and review articles. This part of the scientific literature typically addresses the specific questions of experts rather than those of novices, which need a step-by-step introduction.

Critical assessment The strong emphasis on practical aspects of CSIA together with their systematic presentation makes this book a highly welcome resource for CSIApractitioners. Thanks to the detailed description of typical instrumentation, its correct operation, and possible pitfalls, the information in this book covers all the important points for successful isotope ratio determination. Stable isotope lab managers will be able to review their procedures based on the contents of chapters 3 to 5. Newcomers, in contrast, can find a coherent introduction to a sophisticated piece of instrumentation together with a consistent presentation of the basic terminology.

The more fundamental knowledge is shown together with practical recommendations, which is particularly valuable for non-expert readers. The illustrative topics and applications of Chapter 6 are well-suited to make a case for stable isotope analysis and an excellent motivation for future contributors to CSIA. This part of the book can also provide guidance to lecturers in search of suitable examples for their stable isotope classes.

Summary and readership recommendation Compoundspecific Stable Isotope Analysis is presumably the first book to make this advanced technique accessible to a broader audience. The book is an excellent support for isotoperelated laboratory courses on $\mathrm{MSc}$ and $\mathrm{PhD}$ student level, where it will be used for practical advise as well as for background reading. Experienced researchers and science consultants will appreciate having this book on their shelves as a quick reference to a versatile and developing analytical technique. 